\section{New Bands and Lines in the Twilight Sky Spectrum}

At the Abastumani Astrophysical Observatory we have been making continuous observations of the twilight sky for seventeen years, with the view of studying the features of the Earth's atmosphere ${ }^{1}$. In 1957 , we began to study the twilight sky spectra by means of a grating spectrograph (grating $135 \times$ $90 \mathrm{~mm}^{2}, 1,200$ lines $/ \mathrm{mm}$. ; camera, $f=70 \mathrm{~mm}$., dispersion $80 \mathrm{~A} . / \mathrm{mm}$. in the red region). During the first positive system (mainly with the sequence $\Delta \nu=3$ ). Further, we have found two bands of a system which we believe was not hitherto known. These bands were identified recently by Le Blanc, Tanaka and Jursa ${ }^{2}$ in a laboratory.

The line $\lambda_{\text {obs }}=6707 \cdot 1 \pm 0.5$ observed by us probably corresponds to the line $\lambda_{\text {ubs. }}=6708 \pm 1 \cdot 5$ discovered by Delannoy and Weill ${ }^{3,4}$ in the twilight spectrum. These authors identify this emission with the resonance line of $\mathrm{Li}(6707 \cdot 86 \mathrm{~A}$.). We regard this interpretation as probable, but apparently on our

Table 1

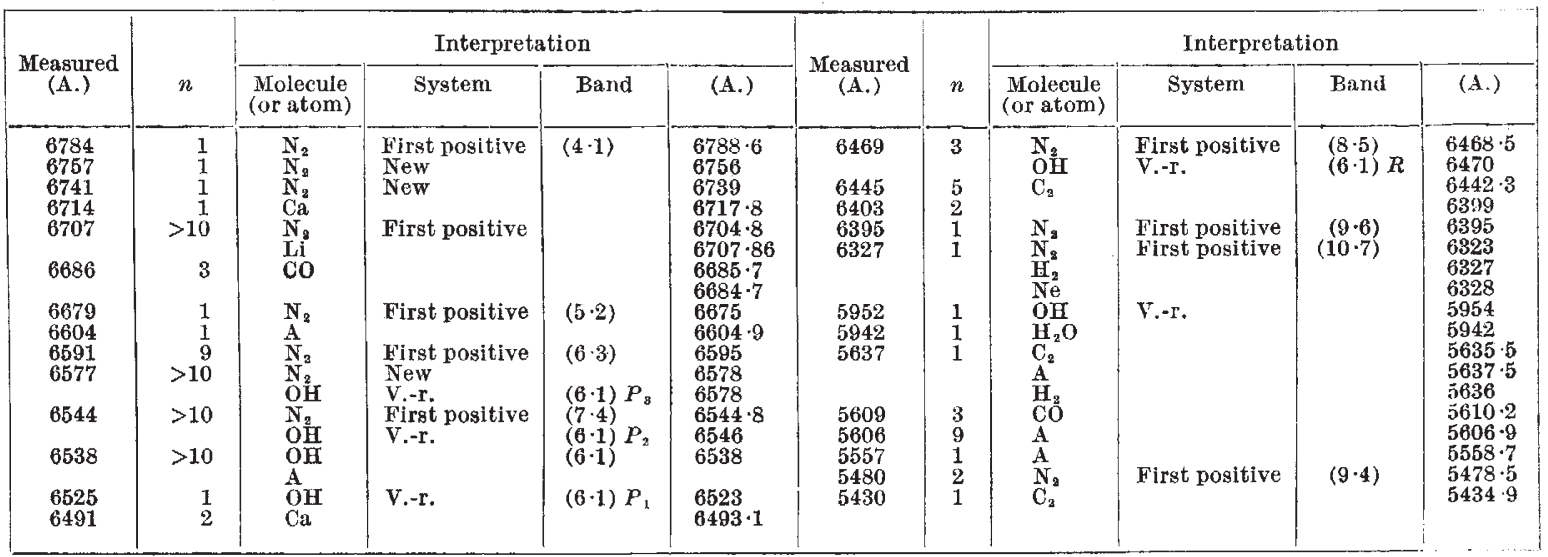

year beginning December 1957,67 spectrograms were obtained in the region 5400-6800 A., of which 29 belong to the morning and 38 to the evening twilight. The well-known lines of oxygen, $\lambda \lambda 6300 \rightarrow 6364 \mathrm{~A}$. [O I], $5577 \mathrm{~A}$. [O I] and the sodium D-lines 5890-5896 A., are always present on these spectrograms. In addition, some new lines and bands appear perman. ently or episodically on them. Two such spectrograms are reproduced in Fig. 1 . Table 1 contains the wave-lengths of the lines and bands ( $\left.\lambda_{\text {obs. }}\right)$, the number of cases of their appearance $(n)$ and the suggested interpretation. This is a preliminary interpretation in so far as in some cases the difference $\Delta \lambda=\lambda_{\text {obs. }}-\lambda$ ( $\lambda$ is an accurate value of the line or band wave-length for a given atom or molecule) reaches the value of 4-5 A., which exceeds the probable error of measurement of $\lambda_{\text {obs. }}(1-2$ A.). Some additional systematic errors in the determination of $\lambda_{\text {obs. }}$ may have occurred. They may be caused by the high intensity and the complicated structure of the continuous spectrum of the twilight sky, on the background of which one has to study faint lines and bands.

There are some bands of molecules of $\mathrm{N}_{2}$ and $\mathrm{OH}$ in Table 1. $\mathrm{N}_{2}$ is presented by the bands of the

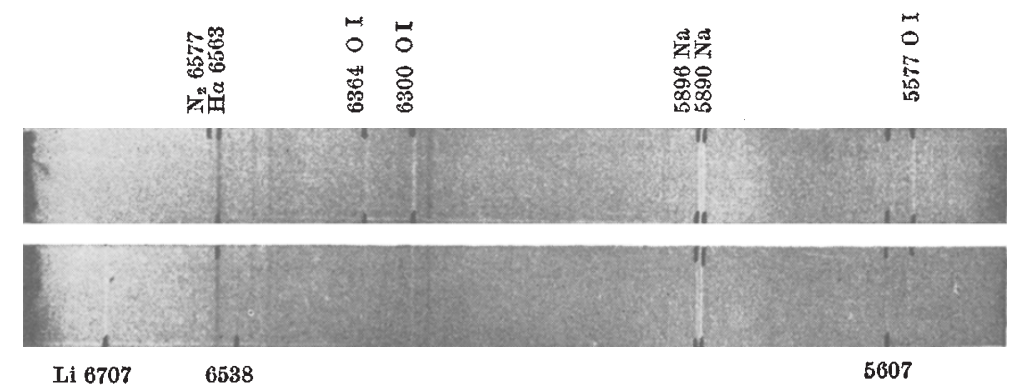

Fig. 1. (a) Augus 22, 1958, $Z=97^{\circ} 33^{\prime}-101^{\circ} 46^{\circ}$. (b) November 11, 1958. $Z=98^{\circ}-101^{\circ} 30^{\prime}$ spectrograms the line fuses with the band $\mathrm{N}_{2}=$ $6704 \cdot 8 \mathrm{~A}$

Our observations are continuing. We intend to publish a fuller account of the observations and particularly to provide information on the height of the layers in which the glow mainly arises.

I. A. Khvostikov

T. G. MEGRELISHVILI

Abastumani Astrophysical

Observatory,

Mount Kanobili,

Georgia, U.S.S.R.

Jan. 16.

${ }^{1}$ Megrellshvili, T. G., and Khvostikov, I. A., Rep. Acad. Sci, U.S.S.R. (Doklady), 59,7 (1948) Megrellishvili, T. T., Bull. Abastumani Astrophys. Ob8., 9 (1949); Rep. Acad. Sci., U.S.S.R. (Doklady) 116,5 (1957).

${ }^{3}$ Le Blanc, F., Tanaka, Y., and Jursa, A., J. Chem. Phys., 28, $N$ 5, 979 (1958).

${ }^{3}$ Delannoy, J., and Weill, G., C.R. Acad. Sci., Paris, 247, 806 (1958),

4 Barbier, D., Delannoy, J., and Weill, G., C.R. Acad. Sci., Paris, 247. 886 (1958).

\section{Density of the Upper Atmosphere}

AN important part of the International Geophysical Year Satellite Programme is the use of satellite orbital data for the deduction of atmospheric densities at very high altitudes. Several reports on preliminary results obtained by

(a) this method have recently appeared $^{1,2}$.

Some years ago, I undertook an analysis of the density of

(b) the atmosphere to extreme altitudes ${ }^{3}$ for use in connexion with certain theoretical studies of extreme-altitude aerodyna- 